\title{
Estudio metalográfico de la calidad del cobre producido en las fundiciones de Cerro Muriano (Córdoba) durante el Período Romano Altoimperial ${ }^{(\bullet)}$
}

\author{
R. Calabrés ${ }^{(*)}$, A.J. Criado ${ }^{(*)}$, J.A. Martínez ${ }^{(*)}$ y J. Storch de Gracia ${ }^{(* *)}$ \\ Resumen El proceso de obtención de un cobre, más o menos puro, depende de gran cantidad de variables y es \\ difícil de controlar. Antes de la romanización, dicho proceso de obtención ya era conocido. \\ Utilizando cobres de primera fusión localizados en un yacimiento arqueológico sito en Cerro \\ Muriano (Córdoba) datados en los siglos I a.C. y I d.C., se ha determinado que sus elaboradores \\ tenían un conocimiento del proceso de fundición de cobre superior al esperado. La existencia de una \\ solidificación celular y celular dendrítica en las muestras estudiadas, así como los análisis realizados, \\ indican una producción de cobre de alta pureza. El estudio permite concluir que, en época romana, ya \\ se conocían los tres procesos básicos de la obtención de cobre: tostación, fusión y escorificación y \\ conversión. Dicho proceso era conocido gracias a la gran experiencia acumulada, aunque estos \\ metalurgistas desconocían las causas que originaban los resultados finales obtenidos.
}

Palabras clave: Metalografía. Cobre. Fundición. Romanización. Siglo I a.C. Siglo I d.C.

\section{Metallographical study of the quality of copper manufactured at the casting installations of Cerro Muriano (Córdoba) during the High Imperial Roman Period}

Abstract The copper obtaining process depends on a great quantity of variables and presents complex
controlling. The mentioned obtaining process was well known since the Roman Age. The knowledge
of copper of the casting process was higher than expected, taking account of the studies with first
melting copper samples localized at an archaeological deposit in Cerro Muriano (Córdoba, Spain)
and dated at the 1st Century B.C and 1st Century A.C. The existence of a cellular and dendritic-
cellular solidification in the studied samples, together with other realized analysis, show a production
of copper with high purity. Present work allows to conclude that the three basic processes on copper
casting (roasting, smelting, slagging and conversion) were well known. This knowledge was due to
the experience, although they did not know the chemical and physical phenomena.

Keywords: Metallography. Copper. Casting. Romanization. 1st Century B.C. 1st Century A.C.

\section{INTRODUCCIÓN}

Era un hecho conocido ya en el período romano, por las referencias de Plinio ( 1 y 2 ) y por otras fuentes escritas de la época (3), que el cobre producido

(•) Trabajo recibido el día 27 de enero de 1995.

(*) Dpto. de Ciencia de los Materiales e Ingeniería Metalúrgica. Facultad de Ciencias Químicas. Universidad Complutense de Madrid. 28040-Madrid (España).

(**) Dpto. de Ciencias y Técnicas Historiográficas, Área de Arqueología. Facultad de Geografía e Historia. Universidad Complutense de Madrid. 28040-Madrid (España). en Cerro Muriano (Córdoba) era de una gran calidad, utilizado, entre otras aplicaciones, para fabricar el famosísimo "cobre de Campania" (4 y 5).

En este estudio se ha utilizado cobre de primera fusión, procedente de derrames, localizado en el yacimiento arqueológico mencionado. Esta fundición debió de realizarse entre los siglos I a.C. y I d.C., atendiendo a otros materiales arqueológicos encontrados en ese contexto.

Además de restos metálicos de fundición, se han estudiado algunas escorias encontradas en el mismo yacimiento, donde se hallan presentes en grandes 
cantidades. Se han obtenido datos interesantes sobre la composición del metal producido y sobre el procedimiento de extracción empleado. Sorprende comprobar la gran pureza del metal y la ausencia de impurezas típicas en fundiciones de cobre.

El estudio de los distintos materiales considerados demuestra un gran conocimiento de los complejos procesos químicos que tenían lugar en el beneficio de los minerales sulfurados de cobre. Con la composición del metal y de las escorias, junto con el conocimiento de la distribución y de la morfología de las diferentes fases componentes, se ha podido proponer, de una forma esencial, cómo era la metalurgia del cobre durante este momento del período romano, sin entrar en la descripción pormenorizada de las operaciones básicas de ingeniería.

Para extraer la información necesaria de las muestras arqueológicas, se ha utilizado microscopía electrónica de barrido (MEB), difracción de rayos $\mathrm{X}(\mathrm{DRX})$, microsonda electrónica (EDS) y absorción atómica. La preparación de las muestras ha supuesto el mayor obstáculo, ya que era vital no sólo obtener datos de la composición química de los diferentes microconstituyentes, sino también información sobre la morfología, tamaño y distribución de éstos, además de su naturaleza física, química y su comportamiento mecánico. También se ha pretendido conocer la naturaleza de la interacción de las diferentes interfases de la fundición y de sus consecuencias metalúrgicas.

\section{TÉCNICA EXPERIMENTAL}

Las muestras analizadas estaban constituidas por los siguientes materiales (Fig. 1):

A: Fragmento de fundición de cobre procedente de un derrame (Figs. 2, 3 y 4).

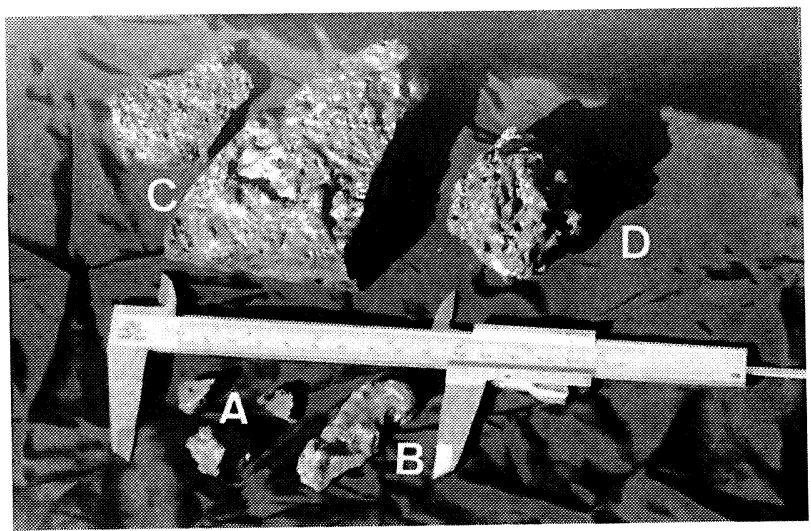

FIG. 1.- Materiales objeto de estudio. Muestras A, $\mathrm{B}, \mathrm{C}$ y D.

Fig. 1.- Studied materials. Samples A, B, C and $D$.

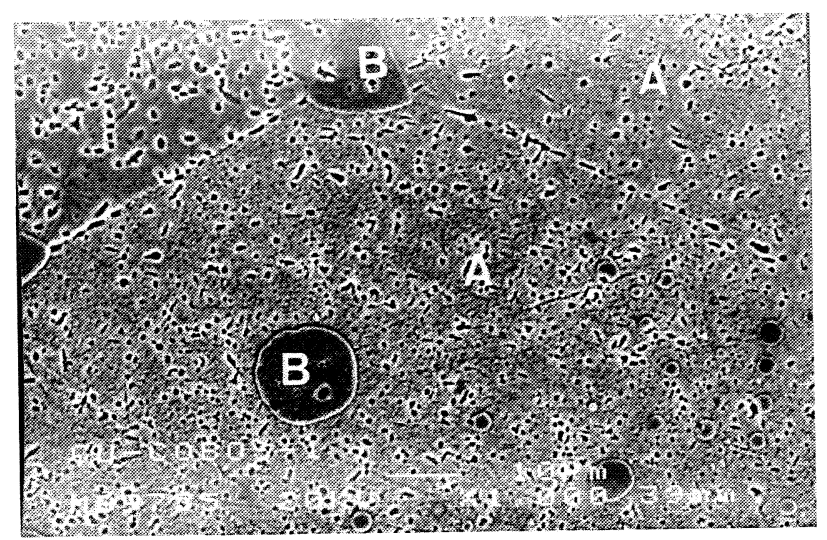

FIG. 2.- Estructura de fundición mostrando una matriz de cobre de granos equiaxiales que contienen inclusiones de sulfuro cuproso (calcosina) situadas preferentemente en límites de grano. Ataque: Nital

$$
10 \%, 60 \mathrm{~s} \text {. }
$$

Fig. 2.-Casting structure showing the copper matrix of equiaxial grains. Droplets of copper sulphide (chalcosine) can be observed preferably at grain boundaries. Etching: Nital 10\%, $60 \mathrm{~s}$.

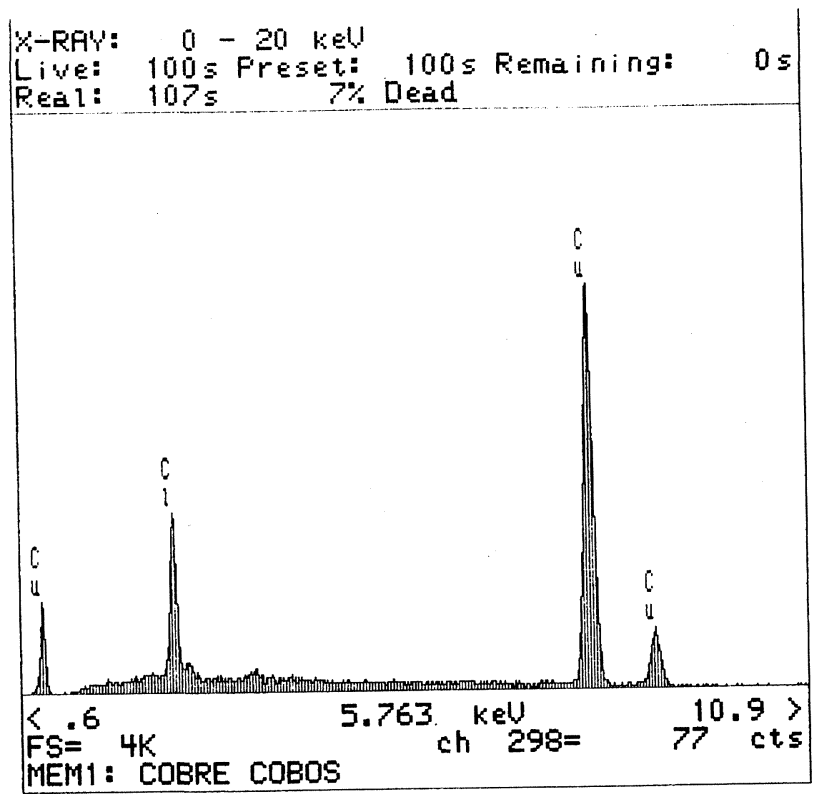

FIG. 3.- Identificación por EDS de la matriz de cobre (A) de la figura 2. La presencia de cloro se debe a residuos del reactivo de ataque químico.

Fig. 3.-EDS identification of the copper matrix (A) from Fig. 2. chlorine presence is due to contamination from the etching.

B: Fragmento de la nata sobrenadante de la fundición (Figs. 5 y 6 ).

C: Escoria que llegó a fundir totalmente (Figs. 7, 8, 9,10 y 11 ).

D: Escoria de fusión incompleta (Figs. 12 y 13).

Estas muestras proceden del yacimiento arqueológico de Cerro Muriano, en la Sierra de Córdoba, a 


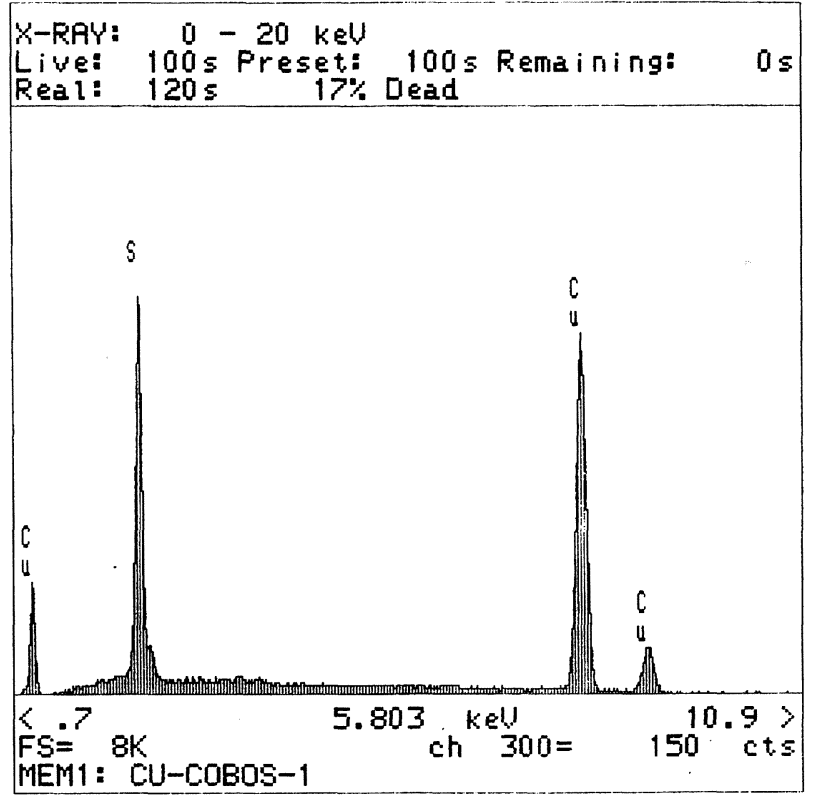

FIG. 4.- Identificación por EDS de las inclusiones de calcosina (B) dispersas en la matriz de cobre de la figura 2.

Fig. 4.-EDS identification of dispersed chalcosine droplets $(B)$ in the copper matrix from figure 2.

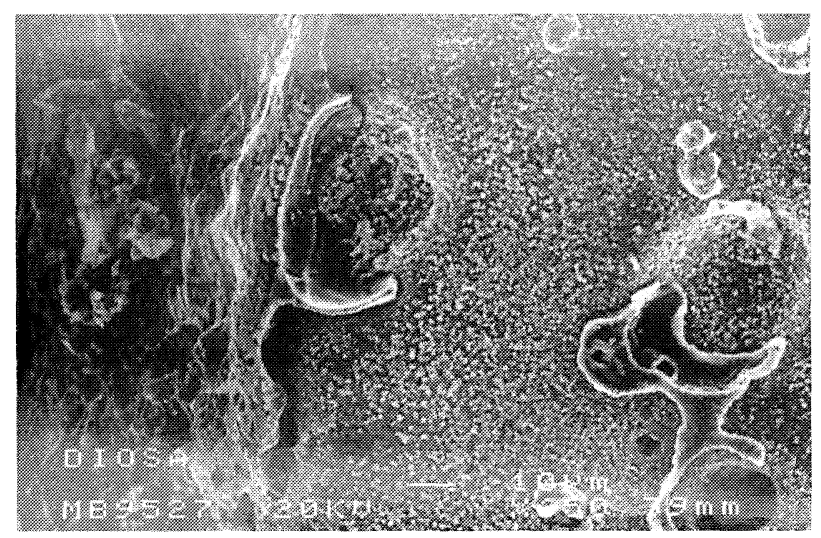

FIG. 5.- Estructura policristalina del cobre de la nata sobrenadante de la fundición. Ataque: Nital $10 \%, 60 \mathrm{~s}$.

Fig. 5.- Polycristalline structure of copper from the skin of the casting. Etching: Nital 10\%, $1 \mathrm{~min}$.

$16 \mathrm{~km}$ al Norte de la capital del mismo nombre (6). Tanto las escorias como las muestras metálicas se pudieron seleccionar y recoger, sin ninguna ayuda técnica, en los extensos escoriales de las fundiciones romanas.

Las muestras descritas se cortaron con un disco de diamante a alta velocidad y para su observación metalográfica se prepararon por métodos convencionales con alúmina $\alpha(0,3 \mu \mathrm{m}), \gamma(0,1 \mu \mathrm{m})$ y magnesia en suspensión ligeramente amoniacal.

$\mathrm{El}$ ataque de las probetas metálicas, procedentes de las muestras A y B, se efectuó con una solución

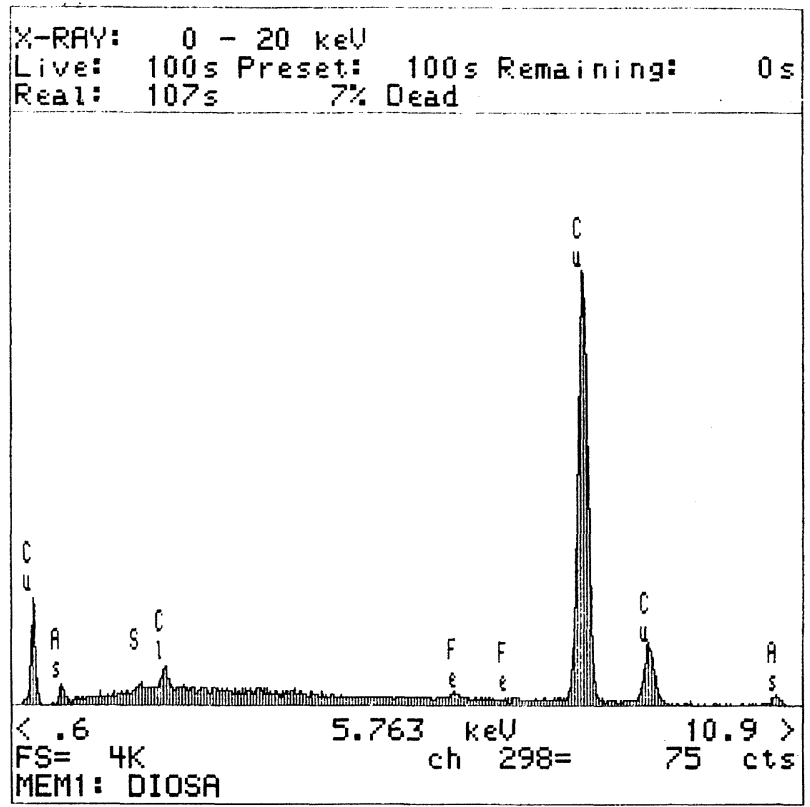

FIG. 6.- Identificación y análisis EDS de la estructura de la figura 5.

Fig. 6.- EDS identification and analysis of the structure shown in figure 5.

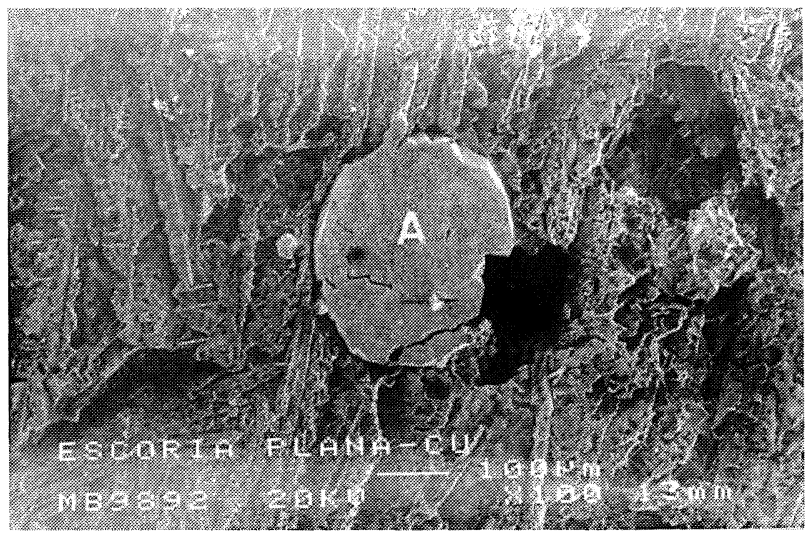

Fig. 7.- Microestructura típica de las escorias del tipo C. Gotícula de cobre con alto contenido de azufre en una matriz de silicato de hierro. Es un testigo de la metalurgia de conversión del sulfuro en cobre metálico. Ataque: HF $45 \%, 2$ min.

Fig. 7.- Typical microstructure of slag C. Droplet of copper with high contents of sulphur in a matrix of iron silicate. This is a core of the conversion metallurgy from sulphide to metallic copper. Etching: $\mathrm{HF} 45 \%, 2 \mathrm{~min}$.

de Nital al $10 \%$ a temperatura ambiente, en baño de ultrasonidos, durante $1 \mathrm{~min}$.

Después de un lavado efectivo, se observaron y estudiaron mediante MEB, DRX, EDS y por absorción atómica.

Las escorias C y D, después de preparadas por el mismo procedimiento descrito para las probetas metálicas, se atacaron con HF en solución acuosa al 


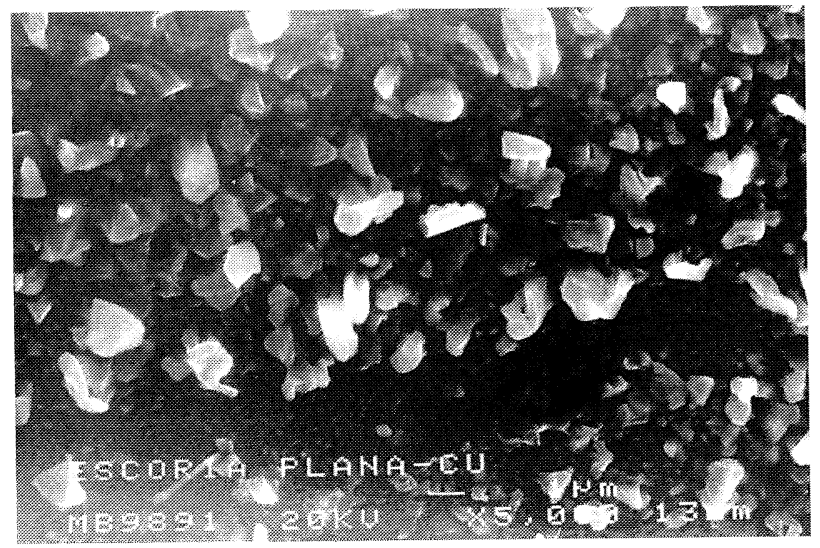

FIG. 8.- Microestructura, a mayores aumentos, de la gotícula de cobre de la figura anterior. Se observa un cobre policristalino con una morfología que es consecuencia de su origen en la etapa de conversión. Ataque: $\mathrm{HF} 45$ \%, 2 min.

Fig. 8.- High magnification structure of the droplet show in figure 7. A polycristalline copper with the morphology consequence of its origin at the conversion stage. Etching: $\mathrm{HF} 45 \%, 2 \mathrm{~min}$.

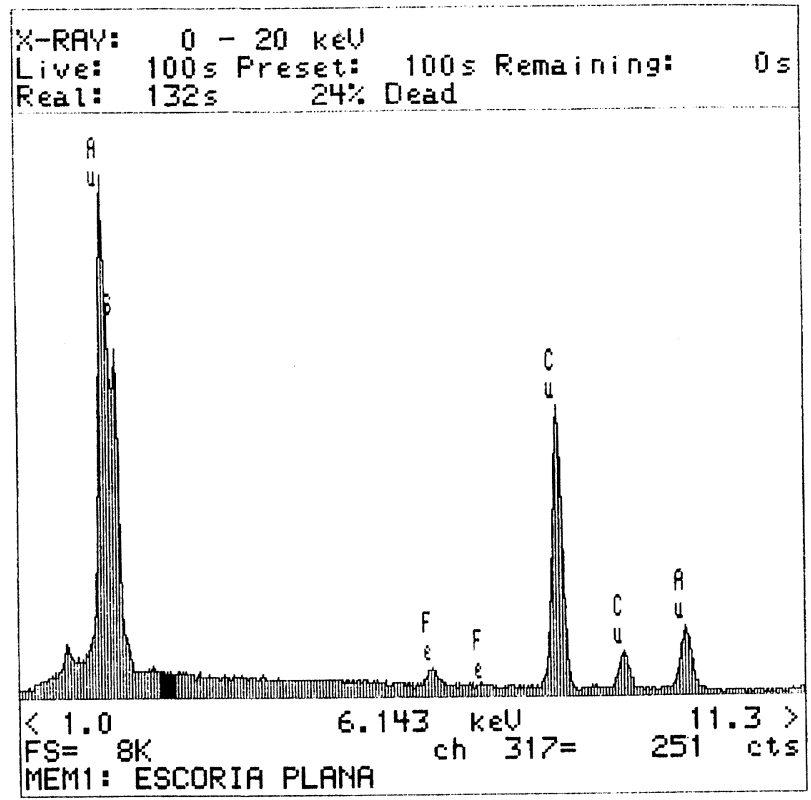

FIG. 9.- Identificación con análisis EDS del material constituyente de la gotícula de las figuras anteriores correspondiente a una escoria tipo $\mathrm{C}$. La presencia de oro se debe al procedimiento de recubrimiento (sputtering) para su observación.

Fig. 9.- EDS identification of the droplet (A) from the preceeding figures. Gold presence is due to the sputtering process of the sample for its observation.

$45 \%$ en masa, durante 2 min, en baño de ultrasonidos. Después de un enérgico lavado, quedaron dispuestas para su examen por los mismos procedimientos que las muestras metálicas.

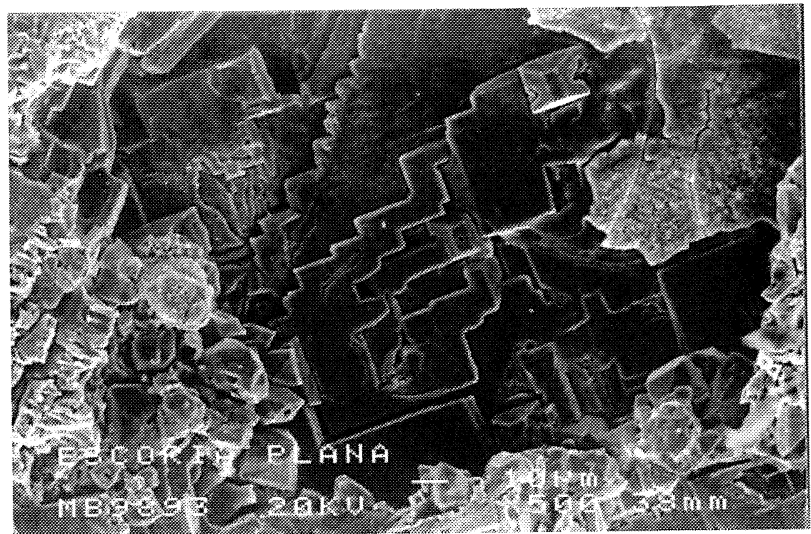

FIG. 10.- Microestructura de silicato de hierro de la escoria derretida tipo C. Ataque: $\mathrm{HF} 45 \%, 2 \mathrm{~min}$.

Fig. 10.- Microstructure of iron silicate from the slag C. Etching: HF $45 \%, 2$ min.

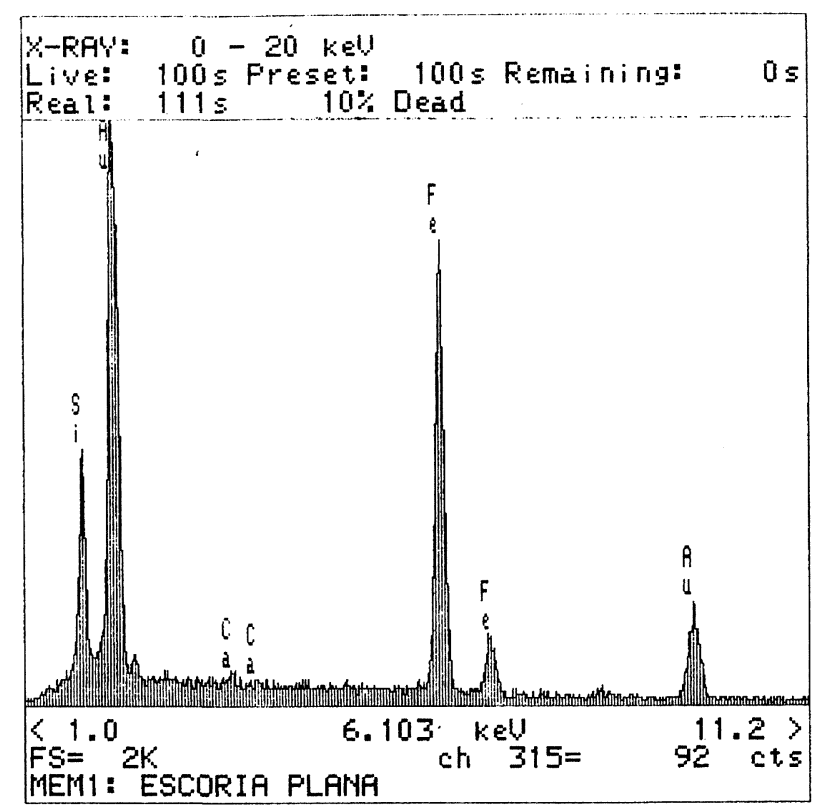

FIG. 11. - Identificación y análisis mediante EDS de la estructura de la figura 10 , que refleja la composición compleja de estas escorias.

Fig. 11.-EDS identification of the structure of figure 10 showing the complex composition of the slags.

\section{ANÁLISIS DE LOS RESULTADOS}

Las probetas del tipo A muestran una matriz de cobre de alta pureza (Fig. 4.), de granos equiaxiales, en la que aparecen unos microconstituyentes de color gris claro, de sulfuro de cobre (Fig. 4) identificados como calcosina, $\mathrm{Cu}_{2} \mathrm{~S}$ (Figs. 14 y 15), situados preferentemente en los límites de grano, con una morfología consecuente con su solidificación en los últimos reductos dejados por el sólido metálico.

La gran pureza de este cobre (Fig. 3) provoca la existencia de una solidificación celular y celular 


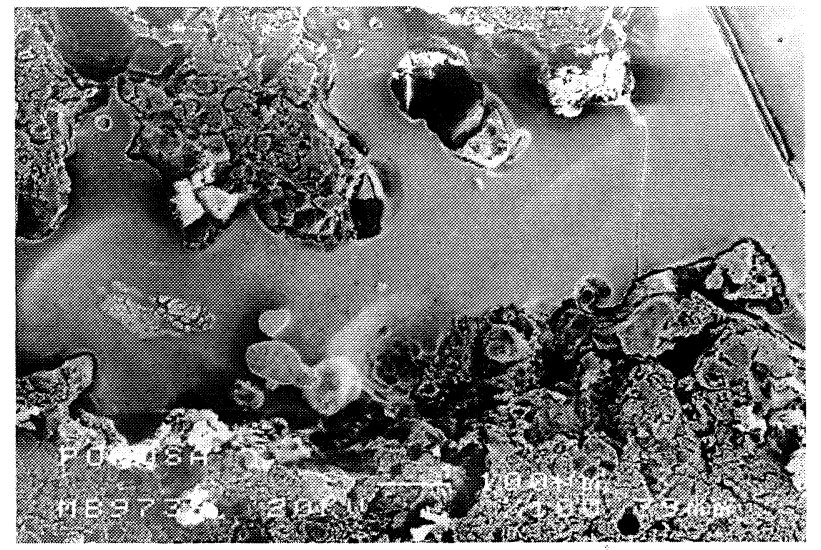

FIG. 12.- Microestructura típica de las escorias poco derretidas del tipo D. Cristal grande de silicato de hierro en una matriz compleja esponjosa del fundente que aparece parcialmente fundida. Ataque: HF $45 \%, 2$ min.

Fig. 12.- Typical microstructure of partially melted D type slags. Iron silicate crystal in a complex swell matrix of fluxing agent. Etching: HF $45 \%, 2 \mathrm{~min}$.

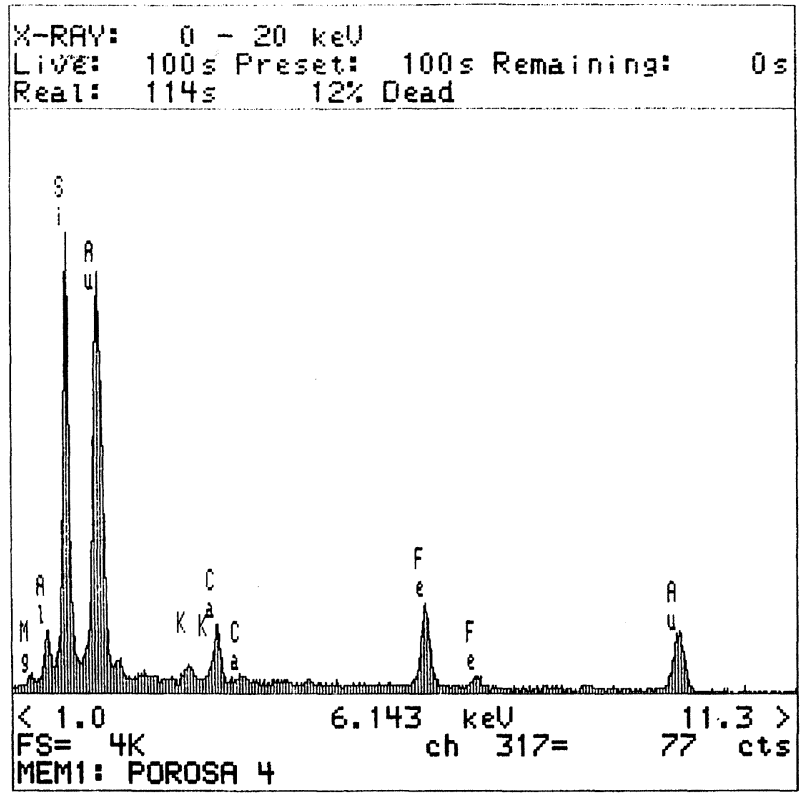

FIG. 13.- Identificación y análisis con EDS del cristal de silicato complejo de hierro de la figura 12.

Fig. 13.- EDS identification of the complex iron silicate crystal from figure 12 .

dendrítica. Posiblemente, la nucleación de este sólido estuvo favorecida por la presencia de impurezas activas, como el hierro, presente en todas las muestras examinadas (6-8).

La estructura de granos equiaxiales, originada en la solidificación de este cobre, demuestra la baja o nula interacción en el sistema de la presencia en el caldo de inclusiones fundidas dispersas de $\mathrm{Cu}_{2} \mathrm{~S}$, probablemente por su propia naturaleza fisicoquí-

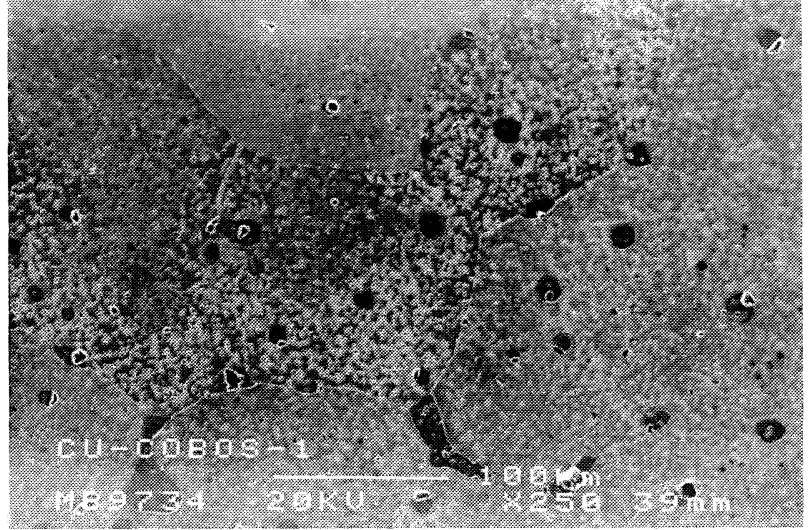

FIG. 14.- Microestructura de la fundición de cobre $\mathrm{A}$, que muestra granos equiaxiales con pequeños cristales de color oscuro constituidos por inclusiones sólidas de calcosina (sulfuro cuproso). Ataque: Nital $10 \%, 1 \mathrm{~min}$.

Fig. 14.- Microstructure of copper casting (A) showing equiaxial grains with small dark crystals of chalcosine. Etching: Nital 10\%, 1 min.

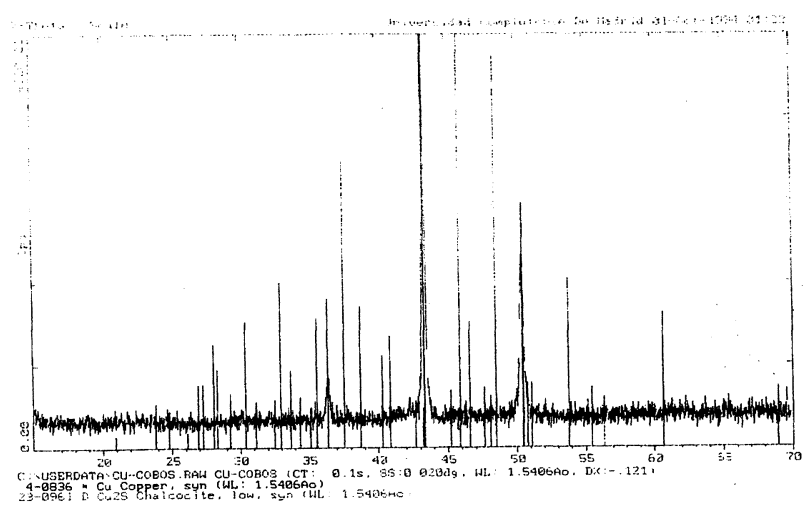

FIG. 15.- Resultados del análisis por DRX de la muestra tipo A. Se observa la aparición de los picos correspondientes a la fase calcosina.

Fig. 15.-X-Ray difracction analysis of sample type A. Picks corresponding to the chalcosine phase are observed.

mica. La existencia de éstas testifica la procedencia mineral, y la pureza de la matriz metálica la secuencia de reacciones químicas seguidas en la metalurgia extractiva de este metal.

La composición de la matriz de cobre refleja un control muy ajustado de los procesos llevados a cabo para la obtención de este metal. Asimismo, la presencia de estos microconstituyentes de $\mathrm{Cu}_{2} \mathrm{~S}$ demuestra una viscosidad alta en el metal fundido, consecuencia de una temperatura insuficiente para permitir su flotación y coalescencia. Estas inclusiones también son testigos de una incompleta oxidación durante el insuflado de aire a la mata fundida, posiblemente, por la precaución de no excederse en la presencia de oxígeno, lo que daría lugar a un metal frágil. 
Es muy posible que en posteriores refusiones y en las operaciones de afino disminuyera considerablemente la presencia de estos microconstituyentes sulfurados. La microestructura de la fundición se muestra con una ampliación de tamaño considerable en las figuras 4 y 5 . La figura 6 recoge los espectros que indican la composición elemental correspondiente al material denominado tipo B, procedente de la nata sobrenadante.

Gracias a esta muestra metálica, se ha podido observar la estructura del cobre solidificando desde una etapa en la que se presenta como un agregado de numerosos cristales idiomorfos, de pequeño tamaño (Fig. 16), a otra en la que por coalescencia de éstos se empiezan a formar grandes cristales de metal (Fig. 17). En ambos casos, la pureza alcanzada es excelente.

Las escorias muestran una información muy valiosa sobre los procesos de obtención de metal seguidos. En ellas quedan las sustancias químicas originadas en las distintas reacciones complejas que tienen lugar durante la reducción.

La presencia de silicatos de hierro en grandes masas vítreas, acompañados de otros componentes en cantidades apreciablemente menores -como los óxidos de este mismo metal-, demuestra que la arena (sílice) fue el fundente utilizado mayoritariamente en estos procesos de fabricación (Figs. 11, 18, 19, 20 y 21). La figura 21 indica restos de metal sulfurado

El sulfuro de cobre, aunque en cantidades reducidas, es un testigo evidente de una etapa de conversión de la mata en cobre, por calentamiento hasta un rango de temperaturas adecuado, y de una oxidación parcial hasta la composición idónea de mezcla de óxidos y sulfuros que, en esas condiciones, dan lugar a un metal de pureza superior al $99 \%$, como se ha comprobado mediante absorción atómica.

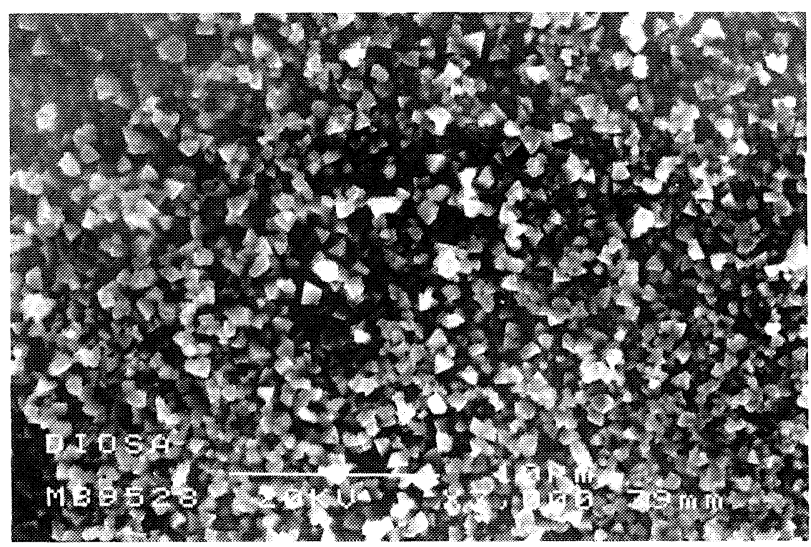

FIG. 16.- Detalle, a más aumentos, de la estructura policristalina de fundición de cobre de la figura 5. Ataque: Nital $10 \%, 1 \mathrm{~min}$

Fig. 16.- High magnification from figure 5. Etching: Nital $10 \%, 1 \mathrm{~min}$.

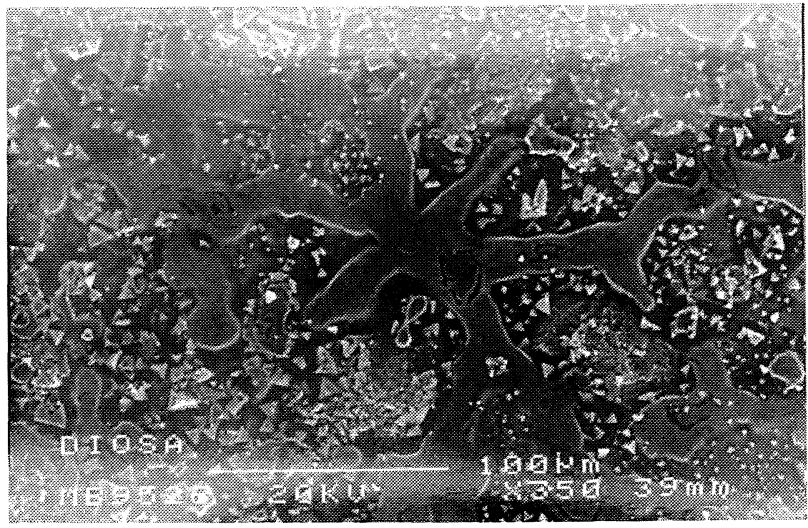

Fig. 17.- Formación de grandes cristales de cobre en el seno de una matriz de finos cristales idiomorfos. Ataque: Nital $10 \%, 1 \mathrm{~min}$.

Fig. 17.- Copper crystal on a matrix of fine idiomorphic crystals. Etching: Nital 10\%, 1 min.

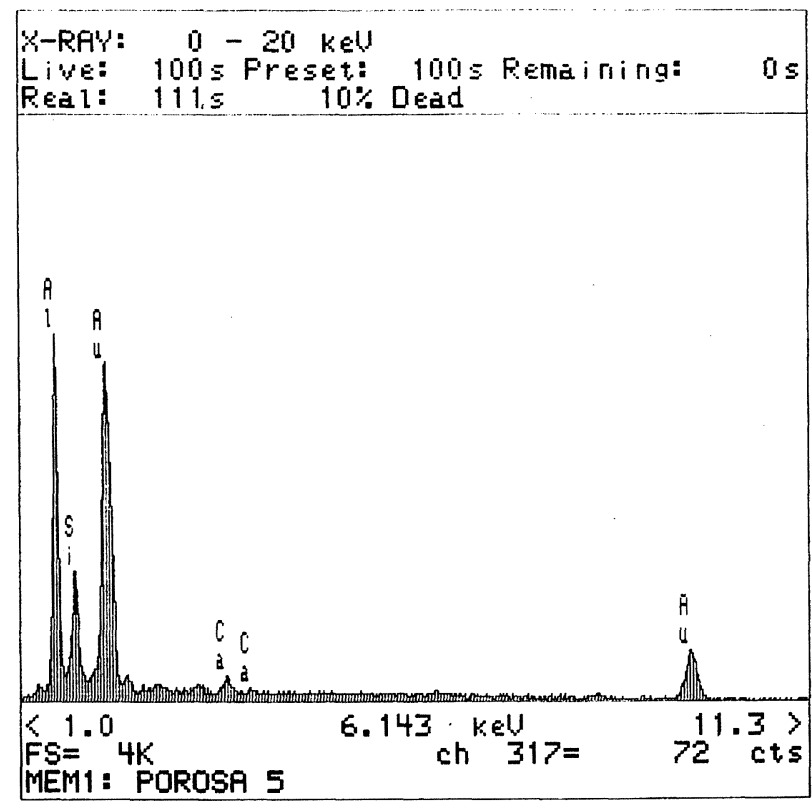

FIG. 18.- Identificación y análisis con EDS de un cristal de silicato complejo de aluminio perteneciente a la escoria poco derretida.

Fig. 18.- EDS identification of a complex aluminium silicate crystal from partially melted slag.

Según se deduce de las observaciones realizadas, el proceso se puede resumir en tres etapas, caracterizadas por las reacciones químicas que suceden en cada una de ellas.

\subsection{Primera etapa: tostación}

El procedimiento para tratar las menas sulfuradas de cobre (calcopirita $=\mathrm{CuFeS}_{2}$ ) es muy 


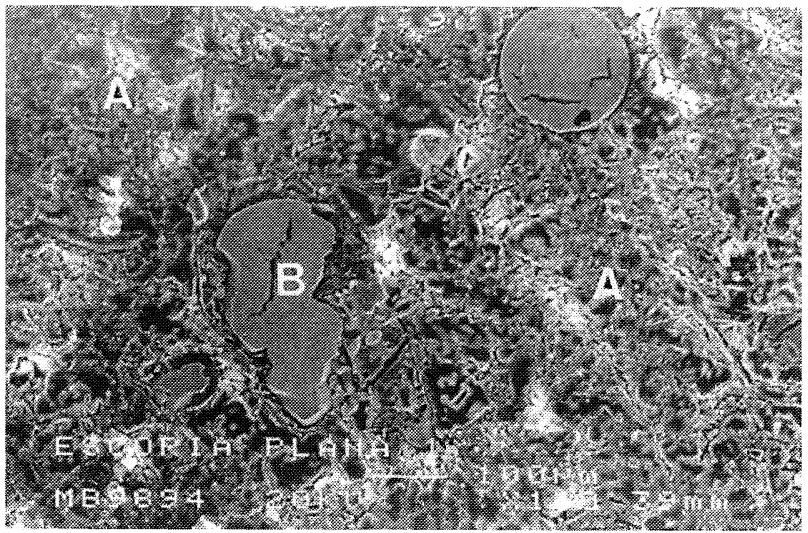

FIG. 19.- Escoria con matriz formada por silicatos de hierro que ocluyen gotículas solidificadas de fundición de cobre. Ataque: HF 45\%, 2 min.

Fig. 19.- Slag with a matrix composed of iron silicates holding droplets of melted copper. Etching: HF $45 \%, 2$ min.

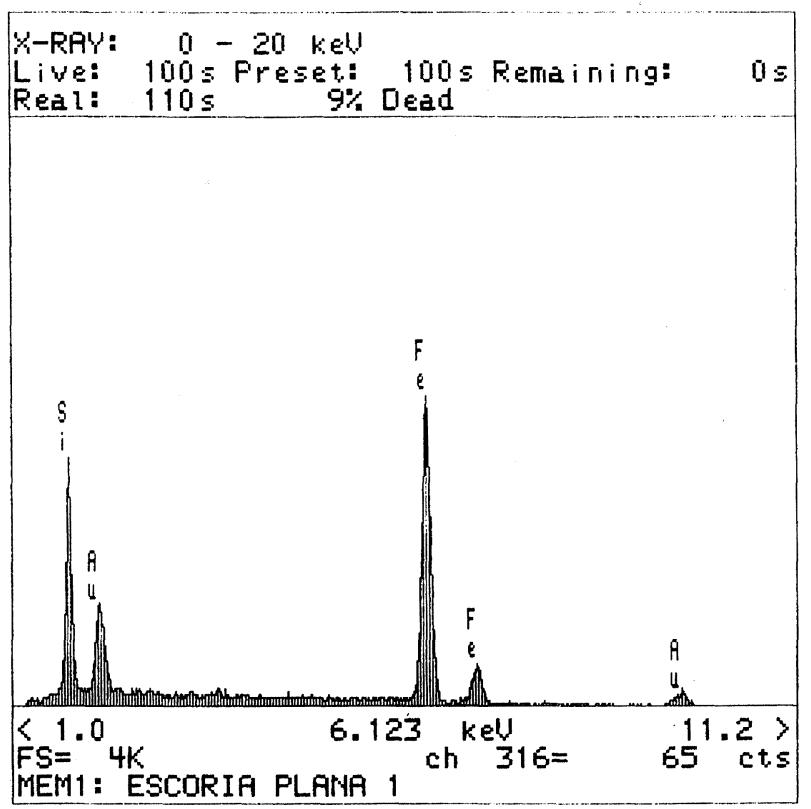

FIG. 20.- Identificación y análisis con EDS de la escoria observada en la figura 19, zona A.

Fig. 20.- EDS identification of slag from figure 19, zone A.

complejo. La obtención del metal afinado necesitaba de varias operaciones, que podían sufrir cambios, para adaptarse a la composición de los minerales de los que se disponía en cada momento. Primero se tostaba la mena (calentamiento en presencia de aire) para desprender el exceso de azufre y la mayor parte del arsénico, antimonio y bismuto, como óxidos volátiles:

$$
\begin{gathered}
4 \mathrm{CuFeS}_{2}(s)+9 \mathrm{O}_{2}(g) \underset{+}{\rightarrow} 2 \mathrm{Cu}_{2} \mathrm{~S}(s)+2 \mathrm{Fe}_{2} \mathrm{O}_{3}(s)+ \\
+6 \mathrm{SO}_{2}(g)
\end{gathered}
$$

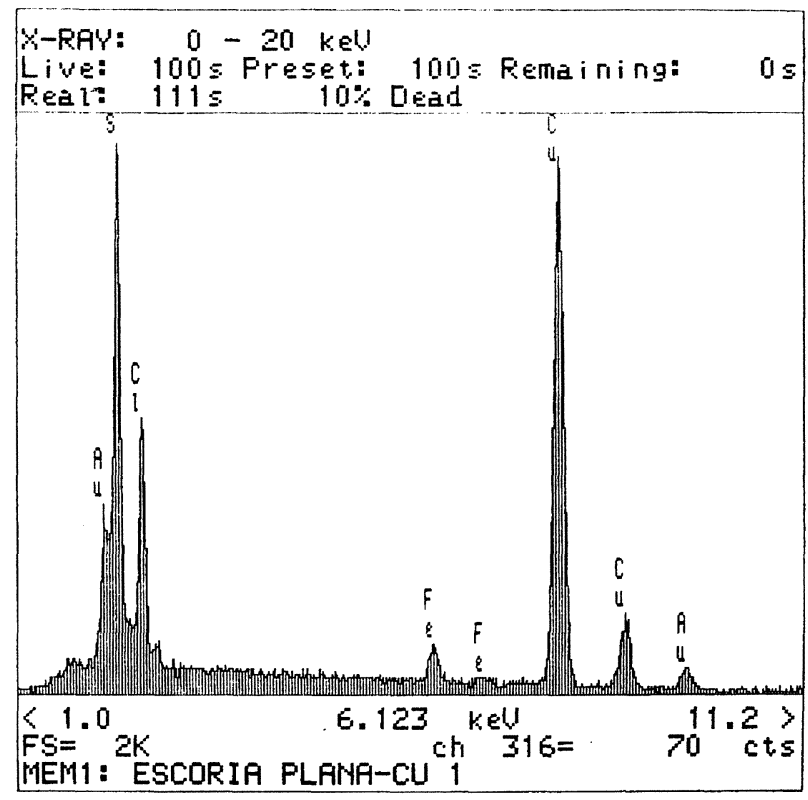

FIG. 21.- Identificación y análisis con EDS de las gotículas observadas en la figura 19, zona B.

\section{Fig. 21.-EDS identification of copper droplets} from figure 19 , zone $B$.

\subsection{Segunda etapa: fusión y escorificación}

Después de tostado, el material se calentaba con carbón vegetal y fundentes silíceos (arena) para llegar a una mata fundida, constituida principalmente por sulfuro cuproso y algunos compuestos de hierro. A través de la mata fundida soplaban aire, oxidando primero al hierro, que así pasaba a la escoria formando un silicato de bajo punto de fusión que se sangraba del horno y se eliminaba por las salidas practicadas a la altura conveniente, según:

$$
\mathrm{Fe}_{2} \mathrm{O}_{3}(s)+3 \mathrm{SiO}_{2}(s) \rightarrow\left(\mathrm{SiO}_{3}\right)_{3} \mathrm{Fe}_{2}(s)
$$

El soplado podría haber sido sustituido por mezclas de carga oxidada constituida por mineral de cobre calcinado totalmente a $\mathrm{CuO}$ con bajo contenido de hierro, o por matas separadas totalmente oxidadas posteriormente.

El componente de la mezcla estaría constituido por matas sin oxidar convenientemente dosificadas.

\subsection{Tercera etapa: conversión}

Una vez eliminada la escoria, se continuaba el soplado hasta que se convertía en óxido la proporción correcta de sulfuro cuproso. El sulfuro y el óxido de la mata oxidada reaccionaban entre sí para proporcionar un cobre de más de un $99 \%$ de pureza, según demuestran los análisis de absorción atómica. 
Sorprende la intuición de aquellos metalúrgicos para ir realizando esta secuencia de operaciones químicas hasta llegar al producto final:

$$
\begin{gathered}
2 \mathrm{Cu}_{2} \mathrm{~S}(s)+3 \mathrm{O}_{2}(g) \rightarrow 2 \mathrm{Cu}_{2} \mathrm{O}(s)+2 \mathrm{SO}_{2}(g) \\
\mathrm{Cu}_{2} \mathrm{~S}(s)+2 \mathrm{Cu}_{2} \mathrm{O}(s) \rightarrow 6 \mathrm{Cu}(s)+\mathrm{SO}_{2}(g)
\end{gathered}
$$

El momento de detener el proceso de oxidación era fundamental para no excederse en la oxidación del cobre y que este resultase muy quebradizo. La señal era -y lo sigue siendo- óptica, por el cambio brusco y llamativo del color de la llama que sale por la boca del horno. Por los resultados obtenidos en nuestros exámenes queda claro que conocían el momento adecuado para la detención del soplado de aire, lo que indica un conocimiento perfecto de la alarma antes citada.

Es muy posible, ya que éste era el procedimiento habitual, que el afino se llevara a cabo posteriormente a las 3 operaciones agitando con palos de madera verde, que al desprender gases reductores rebajaran el contenido de oxígeno hasta valores muy bajos. Este hecho será estudiado cuando se examinen muestras de los lingotes moldeados.

\section{DISCUSIÓN}

Los estudios realizados sobre los materiales recogidos en los escoriales de la minería del cobre de Cerro Muriano (Córdoba), demuestran un conocimiento muy completo de las reacciones químicas que tenían lugar en esta metalurgia extractiva.

Se realizaba una secuencia de operaciones que se ajustaban a las diferentes fases físico-químicas del proceso metalúrgico, consistente en tres etapas: tostación, fusión-escorificación y conversión.

Los silicatos de las escorias, más o menos compactas dependiendo de las temperaturas alcanzadas, demuestran la utilización de arenas silíceas como fundente. En todos los casos existe una retención de pequeñas gotículas de cobre, que son las causantes de que algunas de las escorias, por corrosión atmosférica, adquieran un color verdoso característico. La composición química de las escorias es mayoritariamente de silicato de hierro, conteniendo, en algunos casos, óxidos de hierro, silicatos complejos de aluminio, calcio, magnesio y potasio y la presencia de sulfuros aún sin convertir. La composición química de estas escorias está relacionada con las variaciones de la composición química de las menas utilizadas, con las diferentes gangas que las acompañan, con la mayor o menor eficacia en las operaciones de molienda y concentración de mineral y con las condiciones de operación en los hornos, con una diferenciación clara de las etapas del proceso; entre estas condiciones operativas citare- mos las temperaturas idóneas, características de soplado (oxidación eficaz), la mezcla íntima de los diferentes componentes utilizados y la naturaleza del fundente.

Lo que sí parece estar claro es que todas las escorias recogidas en el yacimiento cordobés proceden de la misma etapa del proceso, propuesta por nosotros como de fusión-escorificación.

Para llegar a la calidad del cobre estudiado, es necesario tostar la mena hasta reducir el contenido de azufre en un $50 \%$ aproximadamente. La separación total del hierro sólo se puede conseguir, en estos procesos pirometalúrgicos, si se incluye una segunda etapa de fusión y escorificación con carbón y fundentes silíceos, después de la de tostación, para obtener una mata y, después por oxidación controlada, eliminar el hierro presente en forma de silicato férrico de bajo punto de fusión, separable fácilmente del horno por sangrado en el momento oportuno.

Sólo con una tercera etapa de oxidación, limitada a la cantidad adecuada de cobre para que se produzca la conversión, es posible llegar a una fundición de cobre de gran pureza como la que se deduce de las muestras estudiadas. Su microestructura es la prueba fehaciente de esta etapa, testimoniada por la presencia de pequeñas inclusiones de sulfuro de cobre no convertidas. Es posible que el control defectuoso de la última fase de conversión, por el color de la llama en caso de oxidación por soplado con aire al elevar su contenido de oxígeno, les llevaría a quedarse algo cortos en la transformación total de la mata obteniendo cobre metálico con óxido cuproso. En todo caso, siempre disponían del recurso de la agitación del caldo, con palos de madera verde, para eliminar el exceso de oxígeno.

Todavía quedan por deducir algunos datos, entre los que sobresalen los referidos a las operaciones de tecnología metalúrgica asociados a las diferentes etapas descritas, de vital importancia para la buena eficacia de las reacciones químicas utilizadas. Se cree después de este estudio, que la calidad del cobre procedente de esta zona y de otras próximas de la sierra y de la campiña de Córdoba, era consecuencia de la composición química de los minerales utilizados, de los fundentes silíceos, del carbón y de un conocimiento profundo de los procesos químicos que tenían lugar en esa metalurgia extractiva. Por tanto, fueron las materias primas y los conocimientos tecnológicos los que condicionaron la excelente calidad del cobre producido en las instalaciones metalúrgicas de Cerro Muriano.

\section{CONCLUSIONES}

- El ataque de las muestras metálicas con Nital al $10 \%$ y de las escorias con solución acuosa de 
$\mathrm{HF}$ al $45 \%$ en baño de ultrasonidos, ha resultado eficaz para su observación metalográfica mediante MEB.

- Los análisis con DRX y EDS han servido para mostrar la pureza de las muestras metálicas examinadas. Asimismo, se ha demostrado una técnica eficaz para la identificación de microconstituyentes en los materiales metálicos y escorias estudiados.

- La presencia de inclusiones de sulfuro de cobre (calcosina) sirve de prueba y testigo para deducir los procesos químicos efectuados en la metalurgia extractiva de este metal.

- La composición de las escorias demuestra la utilización de arenas silíceas como fundente principal, que es eficaz para la eliminación del hierro.

- Se ha observado que la presencia de gotículas de cobre en las escorias depende, en cuanto a su número, del estado que presente la escoria examinada, más o menos derretida, lo que pone de manifiesto la importancia de las temperaturas alcanzadas en el rendimiento en metal para esta metalurgia en las etapas de fusión y escorificación.
- La calidad del cobre examinado está en relación directa con la composición de los minerales y fundentes utilizados, así como con la depurada tecnología romana del momento.

\section{REFERENCIAS}

(1) Plinio, el Viejo. Naturalis Historia, X, 4, 95.

(2) PLINIO, el Viejo. Naturalis Historia, XXXIV, 4.

(3) Corpus Inscriptionum Latinarum. Vol. II: 1.179.

(4) Historia de España, dirigida por D. Ramón MEnÉndeZ PidAl. España Romana. Tomo II, Vol. I: La Conquista y la Explotación Económica, por A. MonTENEGRo y J.M. BLÁZqUEZ. Ed. Espasa-Calpe S.A. Madrid 1982: 366-426.

(5) Criado, A.J. El Cobre Muriano: Símbolo de la máxima calidad en el Imperio Romano. Diario de Córdoba S.A. Enero, 1995.

(6) Hernáez, J., Blázquez, M.L. y Criado, A.J. Prakt. Metallogr., 24, 1987: 119-125.

(7) Hernáez, J., López del Castillo, C., Mellor, B.G., Criado, A.J. y BlázQuez, M.L. Rev. Metal. (Madrid), 25 (5), 1986: 292-295.

(8) Hernáez, J., Blázquez, M.L. y Criado, A.J. Rev. Metal. (Madrid), 17 (2), 1987: 71-77. 